\title{
Revisited: gas-saturated defects in titanium alloys
}

\author{
M.O. Leder, I.Yu. Puzakov, N.Yu. Tarenkova, M.A. Kornilova, K.A. Rusakov \\ PSC «VSMPO-AVISMA Corporation» \\ moleder@vsmpo-avisma.ru, puzakov@vsmpo-avisma.ru, tarenkova@vsmpo.ru, kornilova@vsmpo.ru,
}

\author{
rusakov ka@vsmpo.ru
}

The simulation results of the internal defects generated during deformation by extrusion and rolling are presented herein. It was demonstrated that end-type deformation defects can result in the formation of internal gas-saturated defects not cropping out the surface under the specific deformation conditions. Key characteristics of the generated defects similar to those of the production defects that have been previously classified as metallurgical are determined.

Key words: defect, deformation, discontinuity.

\section{Introduction.}

Gas-saturated defects or «hard alpha» defects are one of the most critical in terms of the quality of defect types detected in titanium and titanium alloy semi-finished products. The presence of such defects in the finished products leads to dramatic loss in their performance which in turn can lead to the most adverse consequences. Therefore, the issue of dealing with the defects is given considerable attention at all production stages. However, for effective dealing with the defects, it is necessary first of all to understand the nature of defect formation.

As a matter of long experience, all gas-saturated defects can be classified by their nature into defects of deformation and metallurgical origin depending on their characteristics.

Deformation defects, i.e. defects generated during deformation, are characterized by asymmetric gas saturation, increased microhardness and presence of multiple discontinuity cropping out the end face or barrel surface of a semi-finished product.

Metallurgical defects, i.e. defects generated during ingot melting, are normally characterized by more symmetric gas saturation, increased or high microhardness and absence of coming out the end face or barrel surface of a semi-finished product.

Sometimes, the defect characteristics do not allow to classify their formation nature clearly, and then the decisive factor is presence or absence of defect cropping out the surface. Absence of the defect cropping out the surface is always interpreted as a sign of its metallurgical nature despite the other characteristics comparable to those of the deformation defects.

To answer the question about the possibility of gas-saturated defect formation during deformation that do not crop out the surface, a process of formation of gas-saturated defects during deformation was simulated and the characteristics of such defects were studied.

\section{Material and experiments.}

The essence of the experiment was to create artificial cracks on the stock end faces followed by deformation and evaluation of semi-products. To evaluate the nature of the crack behavior and define the conditions most prone to the formation of internal defects, the experiment was planned considering different location of artificial cracks relative to the axis of the stock.

A Ø 275x430 mm stock of Ti6Al4V alloy for extrusion followed by rolling was prepared for the experiment. 5 holes with the diameter of $4 \mathrm{~mm}$ and depth of $45 \mathrm{~mm}$ were drilled from both ends of the stock (Figure 1) and rods with the diameter of $4 \mathrm{~mm}$ and length of $42 \mathrm{~mm}$ of the same alloy were tightly inserted into these holes: 
- one hole on the stock axis,

- 2 holes at a distance of $1 / 3 \mathrm{R}$ from the stock axis,

- 2 holes at a distance of 2/3R from the stock axis.

The gaps between the rod and stock metal simulated thin cracks or capillaries which can be formed during deformation in serial production of semi-products (Figure 2).

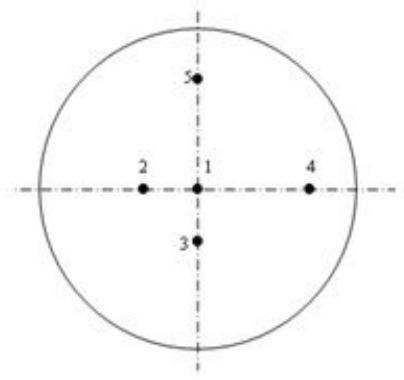

Figure 1. Schematic of hole drilling on the end face of Ø $275 \mathrm{~mm}$ stock

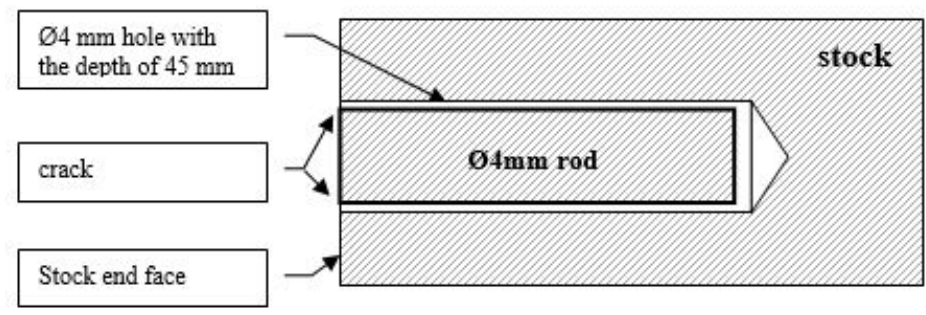

Figure 2. Schematic of artificial crack creation on the stock face end.

The deformation was performed as per serial process by $\varnothing 275 \mathrm{~mm}$ stock extrusion to $\varnothing 152 \mathrm{~mm}$ followed by rolling in $(\alpha+\beta)$-field to $\varnothing 48 \mathrm{~mm}$ and machining to $\varnothing 45 \mathrm{~mm}$. Relative deformation degree is $97 \%$.

The bars were subject to ultrasonic inspection by echo immersion technique, longitudinal wave, with $\varnothing 0,71 \mathrm{~mm}$ FBH, at the background noise level of $25-35 \% \mathrm{FSH}$.

Following the results of bar inspection, the signals with the characteristics shown in Table 1 were detected. The ultrasonic inspection results are schematically shown in Figure 3, where the shaded areas are the areas with USI signals.

Table 1. Ultrasonic inspection results of $\varnothing 45 \mathrm{~mm}$ bars

\begin{tabular}{|c|c|c|c|c|c|c|}
\hline \multirow{2}{*}{ Bar No. } & Template No. & Signal No. & $\begin{array}{c}\text { Defect } \\
\text { size, } \\
\varnothing \mathrm{FBH}\end{array}$ & $\begin{array}{c}\text { Location from the end } \\
\text { face, } \mathrm{mm}\end{array}$ & $\begin{array}{c}\text { Depth, } \\
\mathrm{mm}\end{array}$ & $\begin{array}{c}\text { Length of discontinuity, } \\
\text { mm }\end{array}$ \\
\hline \multirow{3}{*}{$\begin{array}{c}1 \\
\text { (first bar) }\end{array}$} & $1-1$ & 1 & 1,13 & $210-400 \mathrm{~mm}$ & 12 & 190 \\
\cline { 2 - 7 } & $1-2$ & 2 & 1,6 & $0-160 \mathrm{~mm}$ & 17 & 160 \\
\cline { 2 - 7 } & $1-3$ & 3 & 0,67 & $400-780 \mathrm{~mm}$ & 12 & 380 \\
\hline $\begin{array}{c}1-1 \\
\text { (shrinkage } \\
\text { bar) }\end{array}$ & $1-1-1$ & 4 & 0,6 & $1100-1120 \mathrm{~mm}$ & 23 & 20 \\
\cline { 2 - 7 } & $1-1-2$ & 6 & 1,34 & $800-980 \mathrm{~mm}$ & 8 & 180 \\
\cline { 2 - 7 } & & 7 & 2,26 & $270-500 \mathrm{~mm}$ & 15 & 230 \\
\hline
\end{tabular}


It should be noted that for bar No.1-1, which was a shrinkage bar during extrusion, USI signal is intermittent - no signals were found on a $200 \mathrm{~mm}$ long area.

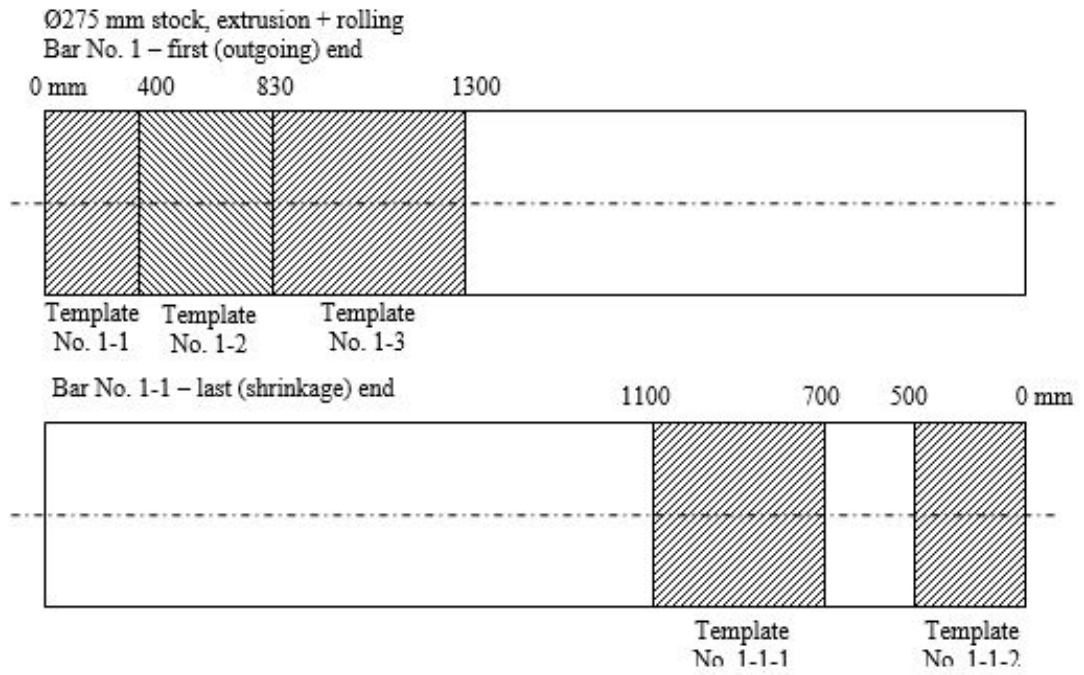

Figure 3. Ultrasonic inspection results of Ø $45 \mathrm{~mm}$ bars.

\section{Results.}

The signals detected during USI were evaluated by macro- and microstructure examination, measurement of microhardness and chemical analysis in several sections to define the nature of morphology change and characteristics of all artificial defects along the rolling direction.

Macrostructure examination of bar No.1 was performed on 18 sections, bar No. 1-1 - on 14 sections.

Figures 4-6 show microstructure photographs of the defects located on the axis, $1 / 3 \mathrm{R}$ and $2 / 3 \mathrm{R}$ of the stock, and detected on bar No.1 during examination of 18 sections. Figures 7-9 show microstructure photographs of the defects detected on bar No. 1-1 during examination of 14 sections. 


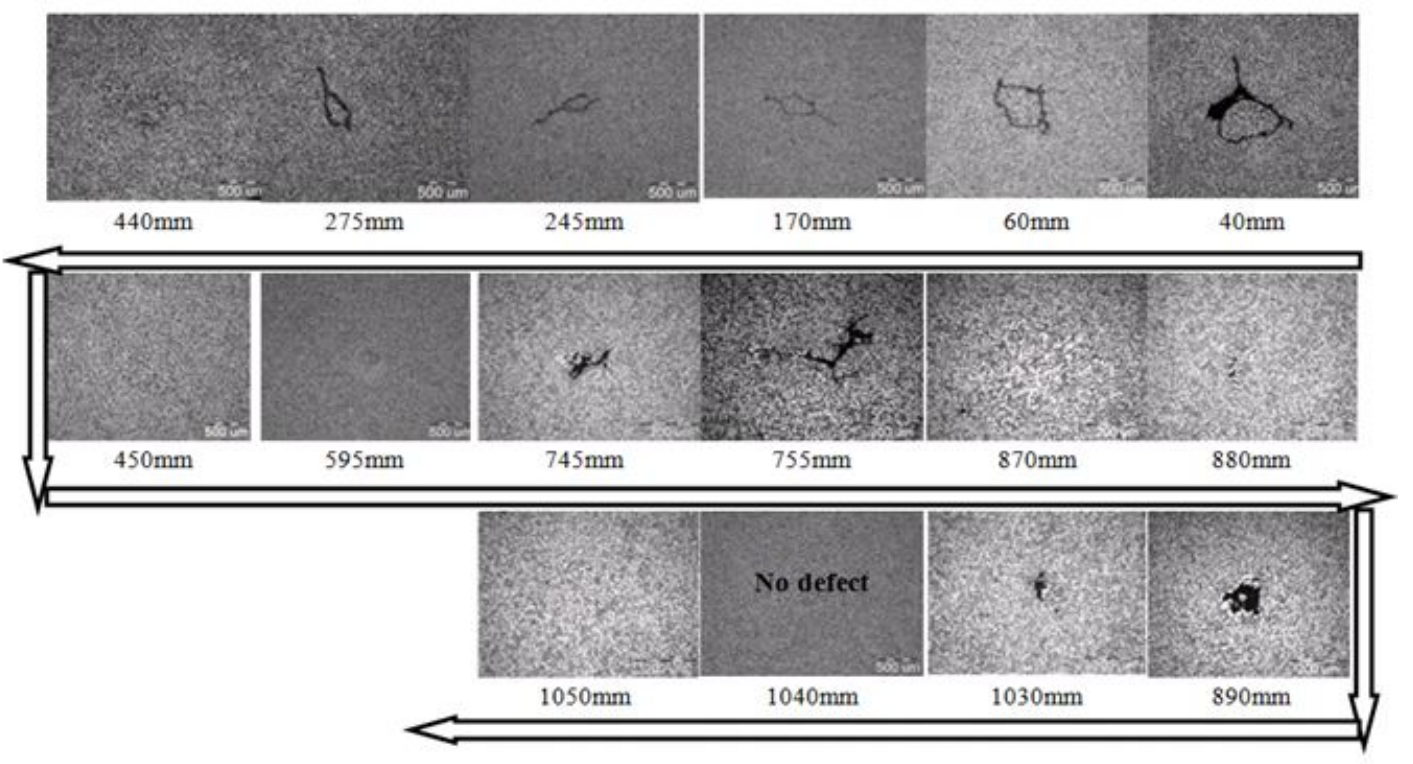

Figure 4. Microstructure photographs of defect No. 1 located along the stock axis, bar No. 1
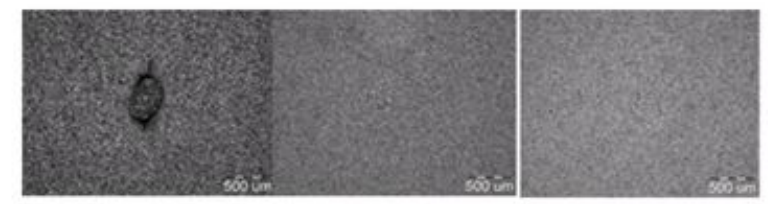

$275 \mathrm{~mm}$

$245 \mathrm{~mm}$

$170 \mathrm{~mm}$
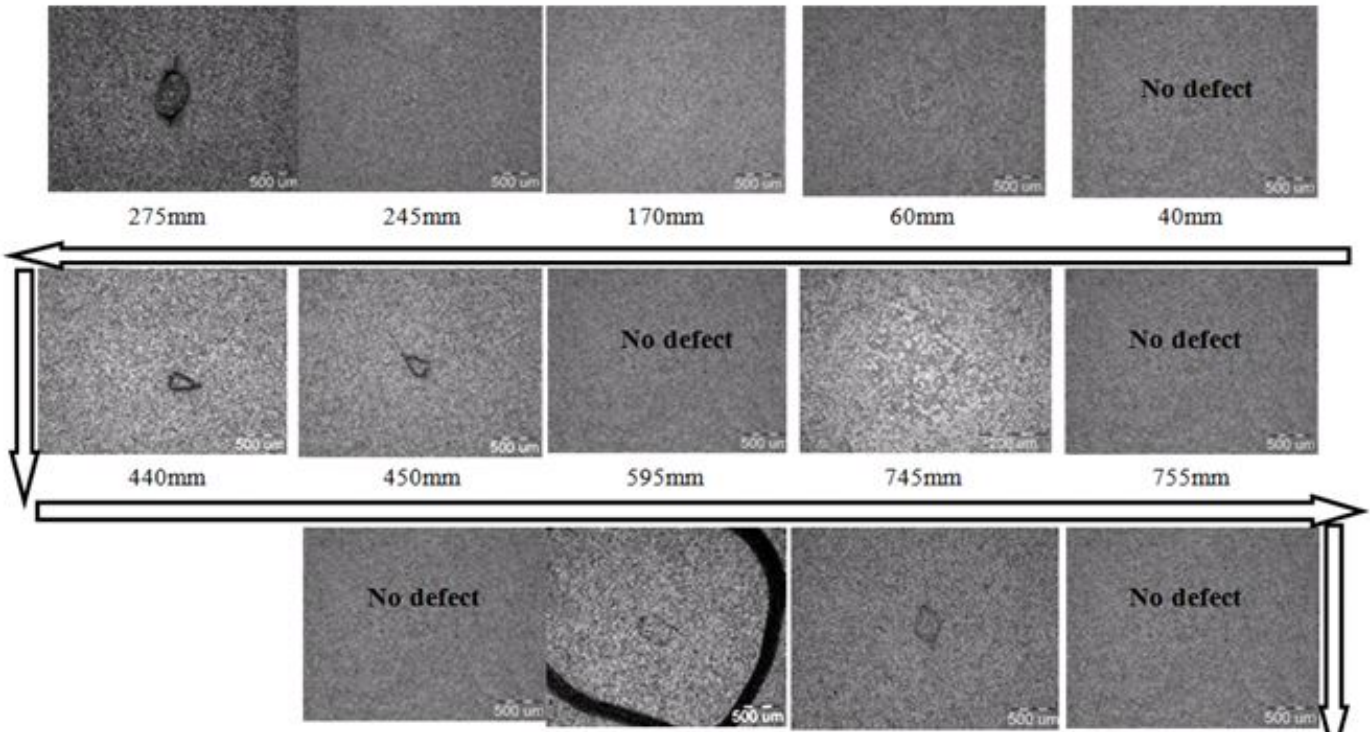

$1030 \mathrm{~mm}$

$890 \mathrm{~mm}$
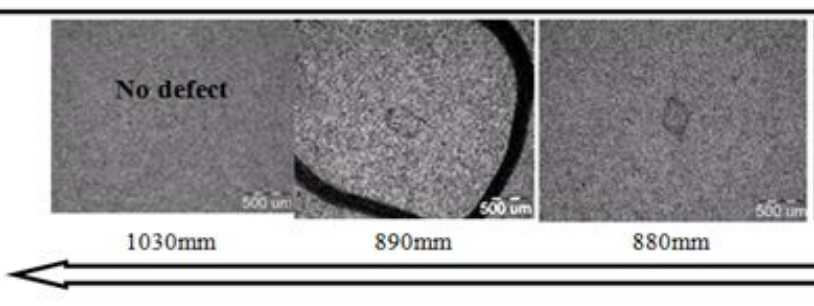

$880 \mathrm{~mm}$

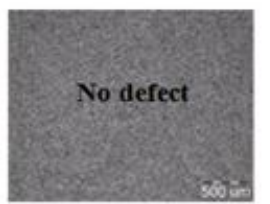

$40 \mathrm{~mm}$

Figure 5. Microstructure photographs of defect No. 3 located on 1/3R of stock, bar No. 1 


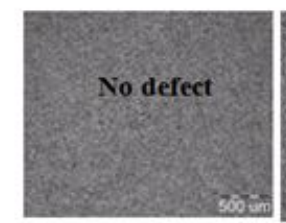

$275 \mathrm{~mm}$

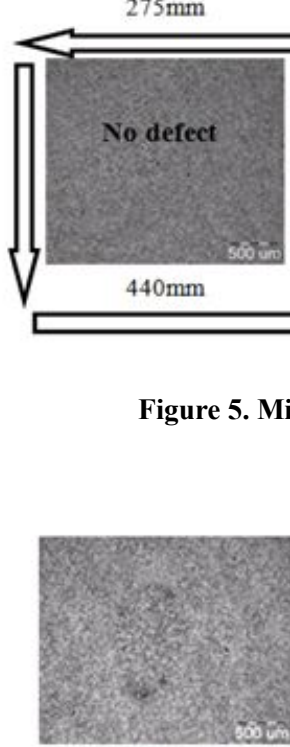

$40 \mathrm{~mm}$

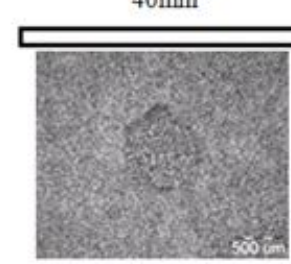

$740 \mathrm{~mm}$

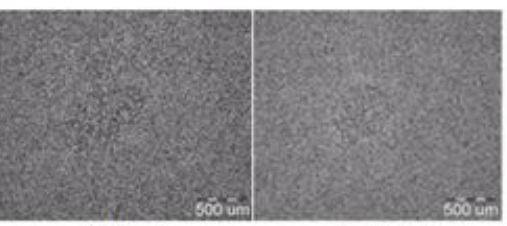

$245 \mathrm{~mm}$

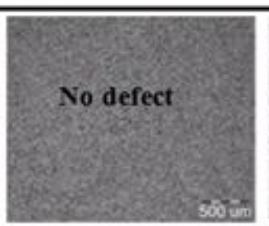

$450 \mathrm{~mm}$

3060

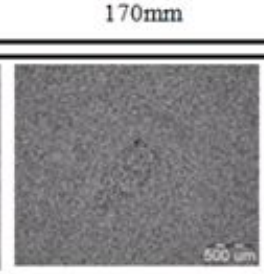

$595 \mathrm{~mm}$

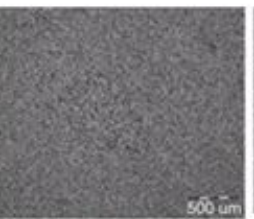

$60 \mathrm{~mm}$

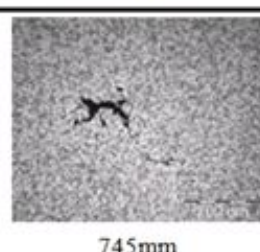

$745 \mathrm{~mm}$

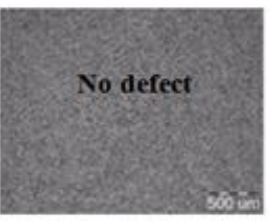

$40 \mathrm{~mm}$

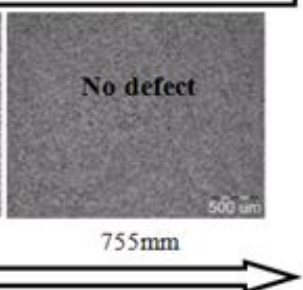

? 


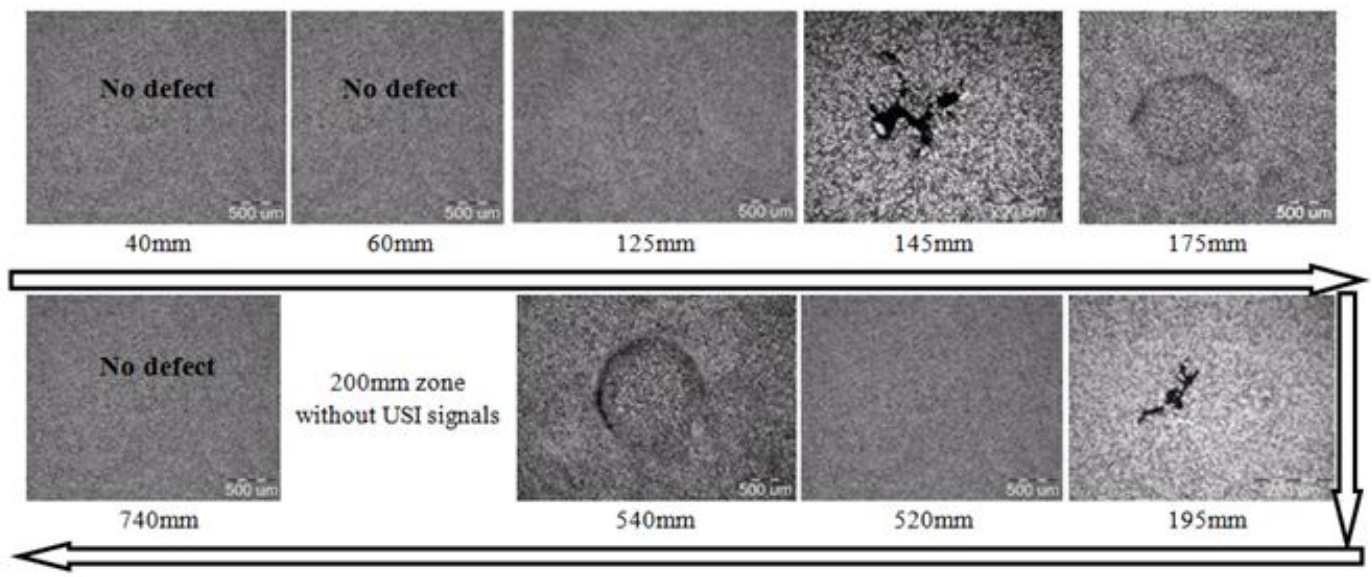

Figure 7. Microstructure photographs of defect No. 3 located on 1/3R of stock, bar No. 1-1

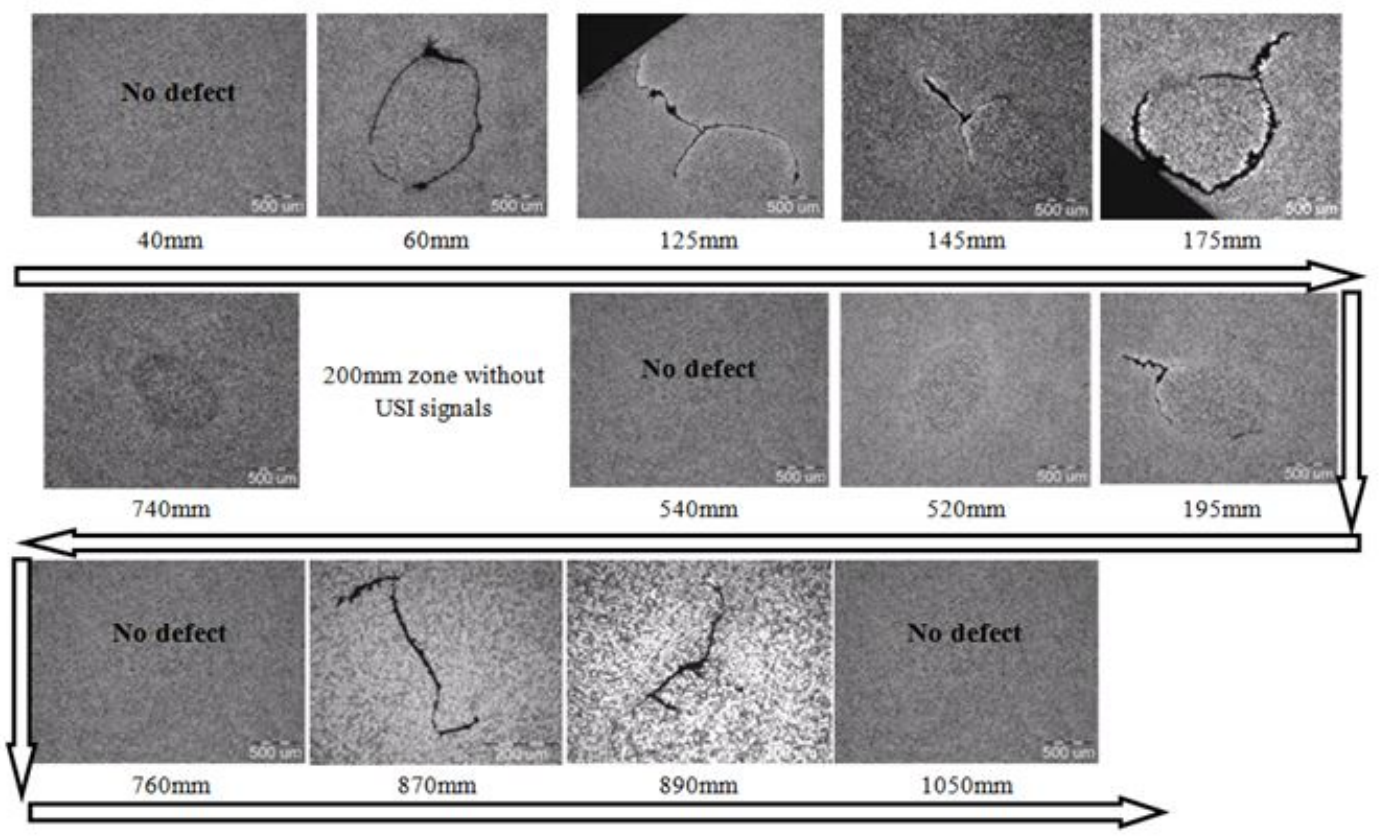

Figure 8. Microstructure photographs of defect No. 4 located on 2/3R of stock, bar No. 1-1

The sequence of the defects found in the axial area of bar No. 1 is of the most interest in terms of microhardness and chemical composition. The summary of the results is graphically shown in Figure 9. 


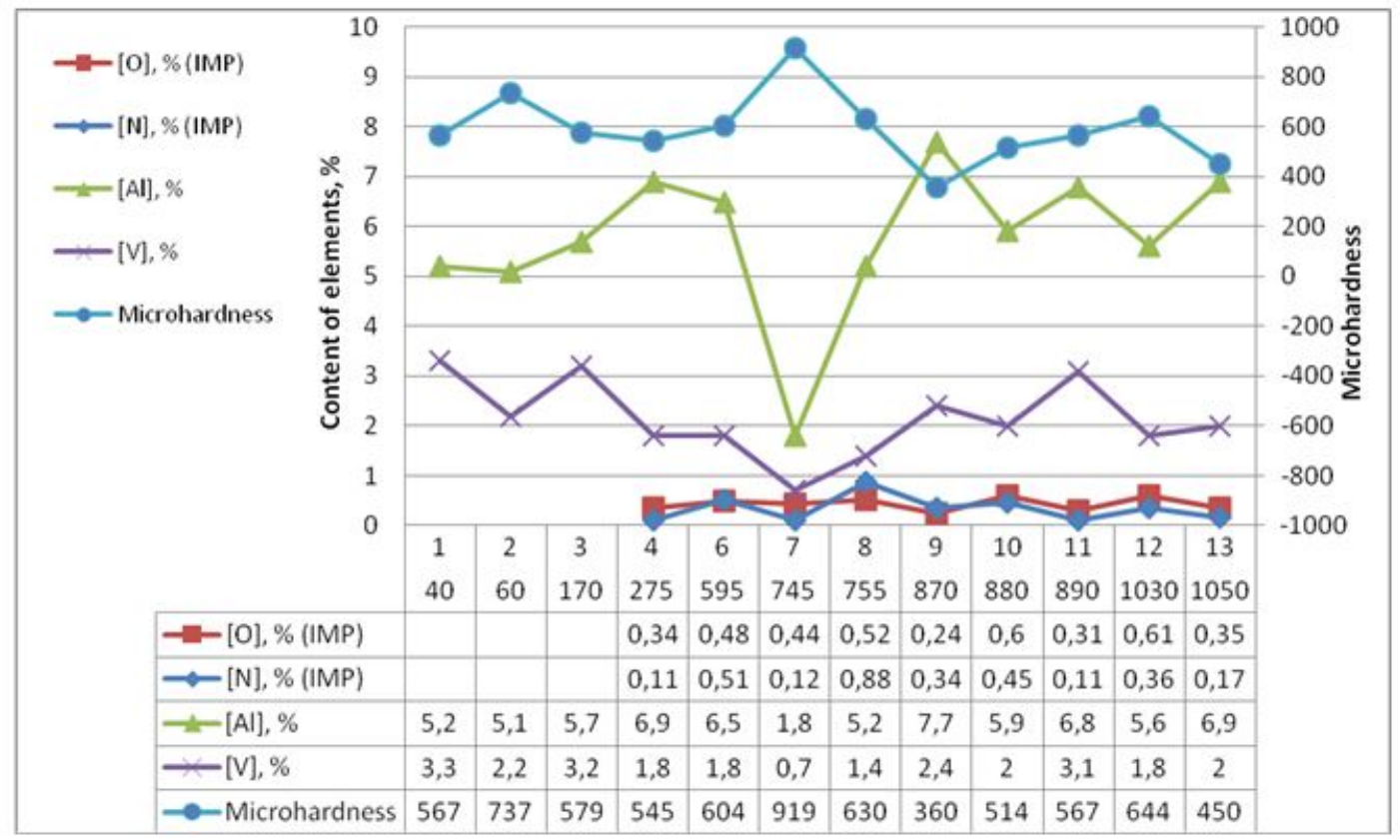

Figure 9. The results of microhardness measurement and chemical analysis of defect No. 1 located along the stock axis, bar No. 1

\section{Discussion.}

The results of macro- and microstructure examination showed that the artificial defects on the stock end face were deformed, stretched in the rolling direction, torn and partially or completely sealed up to the stock metal in several areas during deformation. This is clearly confirmed by sequential arrangement of sections that did not have a defect, and sections in which not only a discontinuity was found but also the defect itself.

Therein, it should be noted that the nature of the artificial defect and parent metal interaction depends on the defect location relative to bar axis and direction of deformation:

- The artifacts located closer to the stock periphery, undergoing a large degree of deformation, have a greater ability to form sealed-up areas.

- The conditions created during metal deformation from shrinkage end contribute to the rupture of artifacts with the formation of internal gas-saturated defects.

The mechanism of formation of defect free areas can be as follows. When heating the stock before deformation operations, the air, oxidizing internal surfaces and forming a gas-saturated layer, gets in the discontinuities artificially created by inserting rods-defects. When deformed, the stock and rod-defect undergo plastic deformation, stretching along the direction of deformation, and gas-saturated layer, that has final size and has no ability to plastic deformation due to high microhardness, is fragmented into parts. In this case, a diffusion welding occurs in the areas free from gas-saturated layer with the formation of locally closed gas-saturated areas.

Thus, during the deformation process, it is possible to create conditions which contribute to the formation of internal gassaturated defects. However, how close will the characteristics of seeded deformation defects be to the characteristics of the production gas-saturated defects classified as defects of metallurgical origin? 
The comparative analysis is given in Table 2. The analysis shows that the characteristics of seeded and production defects are comparable.

Table 2. Comparison of production and seeded defects characteristics.

\begin{tabular}{|c|c|}
\hline Seeded defects & Production defects classified as metallurgical \\
\hline \multicolumn{2}{|c|}{ Microhardness, $\mathrm{HV}$} \\
\hline Up to 919 & Up to 830 \\
\hline \multicolumn{2}{|c|}{ Chemical composition } \\
\hline $\begin{array}{c}\mathrm{A} 1-1,8-11 \% \\
\mathrm{~V}-0,7-7 \% \\
\mathrm{O}-0,24-0,6 \% \\
\mathrm{~N}-0,11-0,88 \% \\
\end{array}$ & $\begin{array}{c}\mathrm{A} 1-1,6-5,3 \% \\
\mathrm{~V}-0,1-2,3 \% \\
\mathrm{O}-0,71 \% \\
\mathrm{~N}-0,78 \% \\
\end{array}$ \\
\hline \multicolumn{2}{|c|}{ Defect length } \\
\hline Up to $1300 \mathrm{~mm}$ from end & $\mathrm{U}_{\mathrm{p}}$ to $1935 \mathrm{~mm}$ from end \\
\hline \multicolumn{2}{|c|}{ Microstructure } \\
\hline 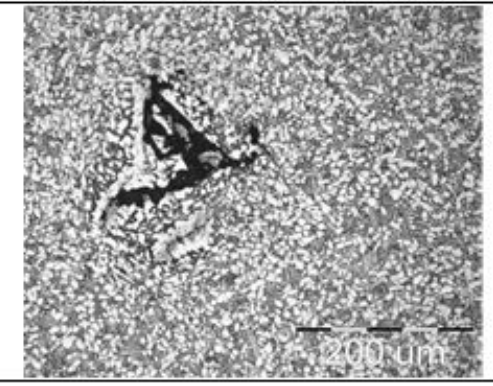 & 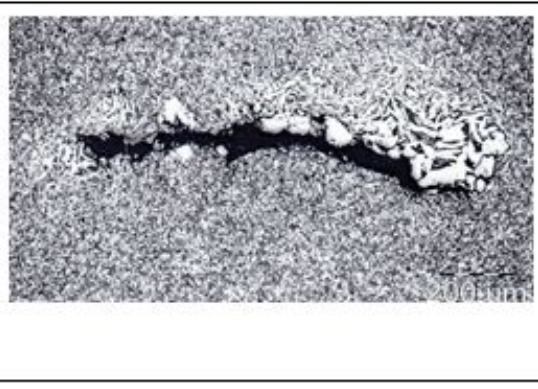 \\
\hline
\end{tabular}

\section{Conclusion.}

1. A process of formation of gas-saturated defects during deformation was simulated and the characteristics of such defects were studied.

2. The obtained results showed that the discontinuities on the stock end face can be closed by deformation with the formation of internal gas-saturated defects that do not crop out the end face.

3. High degree of deformation ( $\sim 97 \%)$ contributed to the diffusion welding of rod-defects with parent metal, not only at the periphery but also along the axis.

4. It is established that the nature of the interaction between artificial defects and parent metal depends on the defect location relative to bar axis and deformation direction:

The artifacts located closer to the stock periphery, undergoing a large degree of deformation, have a greater ability to form sealed-up areas.

The conditions created during metal deformation from shrinkage end contribute to the rupture of artifacts with the formation of internal gas-saturated defects

5. Microhardness and chemical composition of seeded deformation defects are comparable to some production gassaturated defects that have been previously classified as metallurgical based on the study results.

\section{Acknowledgements.}

The authors appreciate E.A. Kalashnik, research engineer of R\&DC Titanium Alloys Laboratory, and V.B. Vykhodets and T.E. Kurennykh, specialists of the Institute of Metal Physics, Ural Branch of the Russian Academy of Sciences, for the invaluable help in the study of defect characteristics. 